Andrea Weiss:

\title{
Bearbeitung von elektronischen Zeitschriften in der Zeitschriftenstelle
}

\section{Vorarbeiten}

Im Frühjahr 2000 begann Frau Mack mit der Bearbeitung der Zeitschriften des Springer-Verlages Heidelberg (ca. 100 Titel), die über den Informationsservice LINK angeboten werden. Diese Titel wurden nach der Freischaltung auf dem Verlagsserver in die EZB eingetragen. Danach übernahm ich im August 2000 für die Zeitschriftenstelle diese Aufgabe.

\section{Allgemeines}

Immer mehr Zeitschriften werden von Verlagen neben der Printausgabe auch als Volltext elektronisch angeboten. Es gibt Abonnementberechnungen für Printausgaben, die bereits kostenneutral auch den elektronischen Zugang zum Volltext enthalten. Diese werden vorrangig bearbeitet. Sinnvoll und rationell ist eine Bearbeitung nach einzelnen Verlagen.

\section{Bearbeitung}

Zur Bearbeitung elektronischer Zeitschriften sind jeweils folgende Einzeltätigkeiten durchzuführen:

- Die Agenturen oder Buchhändler kündigen in Form von Meldungen (z.B. SWETS electronic journal news) oder Verlagsmitteilungen elektronische Volltexte von Zeitschriften an. Diese werden von der Zeitschriftenstelle ausgewertet.

- Die Agentur teilt der WLB auf Anfrage mit, wie man Zugriff auf die Volltexte der Zeitschriften erhält, z.B. direkt über den Verlag oder über Provider (z.B. Ingenta oder Catchword). Dies sind Online-Dienste, die die Volltexte der Zeitschriften auf ihren Servern anbieten.

- Hierfür wird per E-Mail Kontakt mit dem Verlag oder Provider aufgenommen, um den Online-Zugang für den IP-Bereich der WLB zu beantragen und eingerichtet zu bekommen. Dazu ist eine Mitteilung mit Zeitschriftentitel und der Kundennummer notwendig.

- Sobald eine positive Rückmeldung eingeht, wird der jeweilige Zeitschriftentitel in der EZB über eine Administrationsfunktion auf gelb geschaltet (siehe Beitrag über die EZB in diesem Heft) .

- Gleichzeitig muss über ein WWWFormular die lokale URL der Zeitschrift angegeben werden.

- Erst jetzt kann der Benutzer auf die Volltexte zugreifen.

- Nähere Informationen zu den jeweiligen Zeitschriftentiteln oder Verlagen sind über eine Readme-Datei abrufbar (z.B. benötigtes Password, Verlagskonditionen usw.)

- Der Nachweis unseres Bestandes in der ZDB gehört zu den nächsten Aufgaben der KAP.

\section{Derzeitiger Status}

Seit Herbst letzten Jahres wurden ca. 540 Zeitschriften bearbeitet und über die EZB bereitgestellt. Dazu kommen 
weitere 2.713 Zugänge zu kostenlosen Zeitschriften, die auch im Internet über die WLB-Homepage abrufbar sind.

In der Regel werden die letzten aktuellen Jahrgänge im Volltext angeboten, meistens mit Abstracts und in verschiedenen Formaten (HTML- oder PDFDatei). Manche Verlage bieten aber auch größere Zeiträume im Volltext an (z.B. Index to theses, 30 Jahrgänge).

Neben den Zeitschriften des SpringerVerlages sind inzwischen Zeitschriften einiger ausländischer Verlage (z.B. MIT Press, Taylor \& Francis, Oxford Univ. Pr., Blackwell Publ., Cambridge Univ. Pr., Brill), sowie Zeitschriften von badischen Pflichtverlagen wie Wiley VCH in der EZB freigeschaltet worden. Darüber hinaus hat die WLB auch Zugang zu elektronischen Zeitschriften der Verlage Blackwell Science und Munksgaard über eine Beteiligung am SynergyKonsortium.

\section{Dienstleistungen der EZB}

- Über eine mailing-Liste der EZB wird die Zeitschriftenstelle regelmäßig über Änderungen der Zugangsarten für einzelne Zeitschriftentitel informiert, z.B. dass eine Zeitschrift, die bisher lizenzfrei zugänglich war, ab sofort nur noch dann im Volltext zur Verfügung steht, wenn auch die Printausgabe abonniert ist.

- Außerdem werden hier wichtige Informationen und Zugangsbedingungen zu einzelnen Verlagen und Titeln von anderen beteiligten Bibliotheken weitergegeben.

- Die EZB bietet zudem eine Abfragemöglichkeit der monatlichen Nutzungsstatistik.

\section{Benutzerinformation}

In der Zeitschriftenauslage im Lesesaal wurden die Titel, die bereits bearbeitet und elektronisch zugänglich sind, mit einem gelben Schild mit dem Hinweis:

Diese Zeitschrift steht auch als elektronische Publikation zur Verfügung. Bitte wenden Sie sich an die Bibliographische Auskunft auf der Empore gekennzeichnet.

Ebenso wurden die Titel im Kardex mit einem „e“ für elektronisch markiert.

\section{Vor- und Nachteile elektronischer Zeitschriften}

- Der Vorteil bei den Volltexten ist die jederzeitige Verfügbarkeit der Texte, z.B. wenn die gedruckte Ausgabe gerade beim Buchbinder oder entliehen ist.

- Kostenlos zugängliche Zeitschriften können auch vom PC von zu Hause aus übers Internet benutzt werden.

- Es bestehen umfangreichere Recherchemöglichkeiten als beim Printexemplar.

- Nachteile sind z.B. die Beschränkung auf wenige Jahrgänge oder technische Schwierigkeiten (Abhängigkeit von der Verfügbarkeit des Servers) oder geänderte Links der Verlage.

\section{Weitere Vorgehensweise}

- Die weitere verlagsweise Bearbeitung von Zeitschriftentiteln, vor allem aber einiger großer Pflichtverlage (z.B. Thieme), die bereits viele ihrer Titel elektronisch anbieten.

- Die Teilnahme an den jährlichen Anwendertreffen der EZB in Regensburg. 